\title{
Thermodynamics of rate-independent plasticity
}

\author{
G. Puglisi ${ }^{\mathrm{a}}$, L. Truskinovsky ${ }^{\mathrm{b}, *}$ \\ ${ }^{a}$ Dipartimento di Ingegneria Civile e Ambientale, Politecnico di Bari, 70124 Bari, Italy \\ ${ }^{\mathrm{b}}$ Laboratoire de Mechanique des Solides, CNRS-UMR 7649, Ecole Polytechnique, 91128 Palaiseau, France
}

\begin{abstract}
We show that the singular dissipative potential of the phenomenological rate-independent plasticity can be obtained by homogenization of a micro-model with quadratic dissipation. The essential ingredient making this reduction possible is a rugged energy landscape at the micro-scale, generating under external loading a regular cascade of subcritical bifurcations. Such landscape may appear as a result of a sufficiently strong pinning or jamming of defects, leading to elastic micro-metastability. The rate-independent plastic deformation emerges in this description as a continuous succession of infinitesimal viscous events; the limiting procedure presumes the elimination of small time and length scales. We present an explicit example of a simple viscoelastic mass-spring system whose macroscopic dissipative behavior is plastic, rate independent.
\end{abstract}

Keywords: Plasticity; Defects; Rate-independent hysteresis; Dry friction; Thermodynamics; Discrete systems

\section{Introduction}

The phenomenon of rate-independent hysteresis appears in a variety of applications ranging from dry friction to superconductivity (Mayergoyz, 1991).

\footnotetext{
${ }^{*}$ Corresponding author. Tel.: + 33-169333344; fax: + 33-169333026.

E-mail address: trusk@1ms.polytechnique.fr (L. Truskinovsky).
} 
While the phenomenological description of quasi-static dissipative response based on various memory schemes has been rather successful, the microstructural optimization of the hysteretic behavior requires establishing a direct link between the phenomenological coefficients of the macro-models and the microscopic parameters of the material. The main formal difficulty is to connect the macroscopic rateindependent dissipation with the rate-dependent dissipation operative at the microlevel (Onsager and Machlup, 1953), a further task is to relate macroscopic dissipation with the Hamiltonian dynamics at the atomic scales (Berdichevsky, 2003; Kresse and Truskinovsky, 2004).

Many important insights into the problem of bridging the viscous and plastic dissipative constitutive structures at micro and macro scales were obtained in the studies of pinning-depinning dynamics of various types of defects in driven disordered systems (flux lines in type II superconductors, charge density waves in anisotropic conductors, Bloch walls in ferromagnets, contact lines, crack fronts (Fisher, 1998)). Characteristically, all such systems posses a large number of near degenerate metastable configurations and exhibit a cascade of subcritical bifurcations in monotone loading. The rate-independent hysteresis appears as a result of discontinuous dissipative branch switching events known in magnetism as Barkhausen jumps (Bertotti, 1996).

Similar ideas can be applied to the description of rate-independent perfectly plastic deformation understood broadly either as metal plasticity or as transformational plasticity of shape memory alloys (Rice, 1975). The conventional approach reduces to establishing formal rules governing the response to a current load given the history of prior loading. In the case of rate-independent plasticity the macroscopic thermodynamic picture is in contradiction with the microscopic one (Bridgman, 1950; Kestin and Rice, 1970). In particular, the singular behavior of the macroscopic dissipative potential at zero strain rates signals the existence of physical cut-offs.

The goal of the present paper is to show that in order to obtain a conventional macroscopic plastic dissipative potential, represented by a homogeneous function of degree one, from a micro-model with a quadratic (viscous or relaxational) dissipation, it is necessary to homogenize out both small times and small lengths. The main idea, which probably dates back to Prandtl (1928), is to consider rate independence as a limit of rate dependence with zero and unbounded strain rates finely mixed. Experimentalists know that during quasi-static plastic deformation of metals the dislocation dynamics is not continuous but intermittent in space and time with rest periods interrupted by brief moments of energetic activity (Rice, 1970; Zaiser and Seeger, 2002). During these isolated bursts, associated with depinning or nucleation of defect micro-structures, the instantaneous strain rate may exceed the imposed strain rate by several orders of magnitude. Since practically all conversion of mechanical energy into heat takes place during these fast events, the overall dissipation depends only on the number of unstable episodes which is invariant under time re-parametrization. The physical picture behind the quasi-static transformational plasticity is very similar with a replacement of dislocations by phase or domain boundaries (Rosakis and Knowles, 1999; Bhattacharia, 1999). 
In order to develop a prototypical thermodynamical description of rateindependent plasticity consistent with these microscopic observations, it is natural to represent the "jerky" dynamics of the defects as a succession of switching events between neighboring local minima in a sufficiently complex energy landscape where the wells are associated with trapped configurations and ripples represent the barriers between the pinning sites. The evolution inside each energy well can be linked to elastic deformation while the slip can be associated with sudden transition between the wells. In the quasi-static limit the rate effects disappear, but the overall dissipation persists, due to the presence of a sequence of fast transitions between the unstable and stable equilibrium branches. In the context of plasticity a related qualitative pictures have been proposed by Ponter et al. (1979) regarding the activity of Frank-Read sources and by Ortiz (1999) regarding forest hardening. It has also been suggested that strongly interacting defects, constrained to remain on specific crystallographic planes, may form metastable jammed configurations even in the absence of immobile obstacles (Moretti et al., 2004).

To highlight the ideas, we begin with a simple schematization of a defect microstructure as a point (described by a collective coordinate) in an effective energy landscape (see also (Abeyaratne et al. (1996); Choksi et al. (1999) and Deseri and Owen (2002)). We assume that the microscopic dynamics is of the gradient flow (viscous) type and that pinning is sufficiently strong to generate micro-metastability. This brings two dimensionless parameters: $v$ - the ratio of the characteristic times associated with external driving and internal relaxation and $\delta$ - the ratio of the pinning-induced and external length scales. We first show that in the quasi-static limit $v \rightarrow 0$ the dissipation in such systems is localized in a sequence of time discontinuities. To accommodate this effect in the thermodynamic formalism we introduce an integral form of the Gibbs identity which takes into account that the generalized derivative of the free energy is a measure with a non-trivial singular part. We then consider the limit $\delta \rightarrow 0$ when the distribution of the discontinuous dissipative events becomes dense while the dissipation associated with each event tends to zero. In this limit the singular component of the equilibrium stress-strain response disappears and we obtain the conventional constitutive structure of the phenomenological plasticity.

To demonstrate that the phenomenological assumptions of such zero-dimensional model regarding the structure of the energy landscape are realistic, we proceed with a study of a simple one-dimensional mass-spring system generating an equivalent multibranch equilibrium response. This discrete mechanical model simulates a set of rigid atomic planes interacting through bi-stable shear springs; it deals not only with the total slip but also with its spatial distribution even if in a rudimentary manner. The elemental bi-stability reflects the presence of two states - jammed and released; an additional assumption of piece-wise linearity allows for an explicit separation of the motion into adiabatic and jump parts. We focus on the consequences of gradient flowtype dynamics in this system; the equilibrium properties have been previously studied in (Müller and Villaggio, 1977; Fedelich and Zanzotto, 1992; Puglisi and Truskinovsky, 2000, 2002b). To simplify the picture we ignore long range interactions (Truskinovsky and Vaichtein, 2004b), disorder (Sethna et al., 1993), and inertia (Balk et al., 2001). 
It is necessary to mention that viscous augmentation of the phenomenological perfect plasticity (with convex potential) involving Yosida regularization and implying the analog of our limit $v \rightarrow 0$ has been studied earlier by Suquet (1981) and Anzellotti and Luckhaus (1987). The analysis of wiggly energies, implying our limit $\delta \rightarrow 0$, have also been employed previously to obtain the threshold-type kinetics in transformational plasticity (Abeyaratne et al., 1996; Menon, 2002). Our present study, involving the concurrent limits $v \rightarrow 0, \delta \rightarrow 0$, represents an attempt of the synthesis of the above approaches.

The paper is organized as follows. The main ideas are introduced heuristically in Section 2 where we study a "toy" model with a periodic energy landscape. The main purpose of this section is to capture the time behavior of the system while omitting the issues related to the spatial distribution of slip. In the limit of infinitely slow loading and infinitely fine scale of pinning we obtain an explicit description of the macroscopic rate-independent plastic flow and show that the final picture does not depend on the order of the limiting procedures. In Section 3 we consider a more realistic discrete mechanical system whose behavior clarifies the assumptions of the "toy" model and generates specific relations between the macro and micro parameters. A detailed study of the non-equilibrium energy landscape for this model allows us to specify the (gradient flow induced) transformation mechanism and obtain an explicit decomposition of the external work into dissipated and stored energy. We then study in Section 4 an alternative dynamics when the system can escape from a local minimum if the smallest barrier reaches below a given threshold. A sketch of a fully thermodynamical version of the model containing a derivation of the equation for temperature is presented in Section 5.

\section{Heuristic ideas}

Consider an isothermal, spatially homogeneous thermodynamical system characterized by a free energy density $f=f(\varepsilon, \alpha)$, where $\varepsilon$ is the total strain and $\alpha$ is an internal variable representing distributed slip. The second law of thermodynamics requires that

$$
\mathrm{d} w-\mathrm{d} f=\mathrm{d} q^{\prime} \geqslant 0,
$$

where $\mathrm{d} w=\sigma \mathrm{d} \varepsilon$ is the incremental work, $\sigma=\partial f / \partial \varepsilon$ is the stress, $\mathrm{d} q^{\prime}=g \mathrm{~d} \alpha$ is the irreversible entropy production, and $g=-\partial f / \partial \alpha$ is the configurational force (affinity). If the internal relaxation is of the gradient flow type and $\dot{\alpha} \sim g$, the dissipative potential, $D:=g \dot{\alpha}$, is quadratic

$$
D \sim \dot{\alpha}^{2} .
$$

The ensuing model is rate dependent and is not suitable for the macroscopic description of rate-independent plasticity. To be appropriate, the dissipative potential must be a homogeneous function of degree one (Lubliner, 1998):

$$
D \sim|\dot{\alpha}| .
$$


The main problem is how to make the above constitutive structures compatible: gradient flow-type, Eq. (2.2), operating at the micro-level, and plastic-type, Eq. (2.3), operating at the macro-level.

We begin with the microscopic model and assume that a system with quadratic dissipation is driven quasi-statically in a hard device at a constant strain rate $\dot{\varepsilon}=$ $v>0$ (loading). The dynamics is governed by the following system of equations:

$$
\dot{\alpha}=-\gamma \frac{\partial f(\varepsilon, \alpha)}{\partial \alpha}, \quad \dot{\varepsilon}=v,
$$

where the positive parameter $\gamma$ characterizes the time of relaxation. In terms of the slow time $\tau=v t$ (Cardin and Favretti, 2003) we can rewrite the first equation as $\nu \mathrm{d} \alpha / \mathrm{d} \tau=-\partial f / \partial \alpha$, where

$$
v=\frac{v}{\gamma}
$$

is a non-dimensional measure of the external strain rate. If $v \rightarrow 0$ (quasi-static or inviscid limit) the dynamic equations (2.4) can be replaced by a condition of equilibrium $\partial f(\varepsilon, \alpha) / \partial \alpha=0$ defining the stationary locus. In the regular points this procedure allows one to (adiabatically) eliminate the parameter $\alpha=\hat{\alpha}(\varepsilon)$ and to compute the equilibrium free energy $\hat{f}(\varepsilon)=f(\varepsilon, \hat{\alpha}(\varepsilon))$. In the vicinity of a turning point (or a point of subcritical bifurcation) where $\partial^{2} f(\varepsilon, \alpha) / \partial \alpha^{2}=0$ the function $\hat{\alpha}(\varepsilon)$ becomes multi-valued and the dynamical system (2.4) undergoes a transition to a new equilibrium branch in the fast time scale $t$ (Arnol'd, 1994).

Now assume that the system experiences a cascade of branch switching events at regularly spaced internal thresholds. A representative structure of the stationary locus $\hat{\alpha}(\varepsilon)$ is shown in Fig. 1. The thick line indicates the behavior of the dynamical system (2.4) in the quasi-static limit $v \rightarrow 0$. One can see a succession of "slow-fast" transitions, represented as discontinuities, outside which the system remains in the vicinity of one of the equilibrium branches. This scenario describes the "maximum

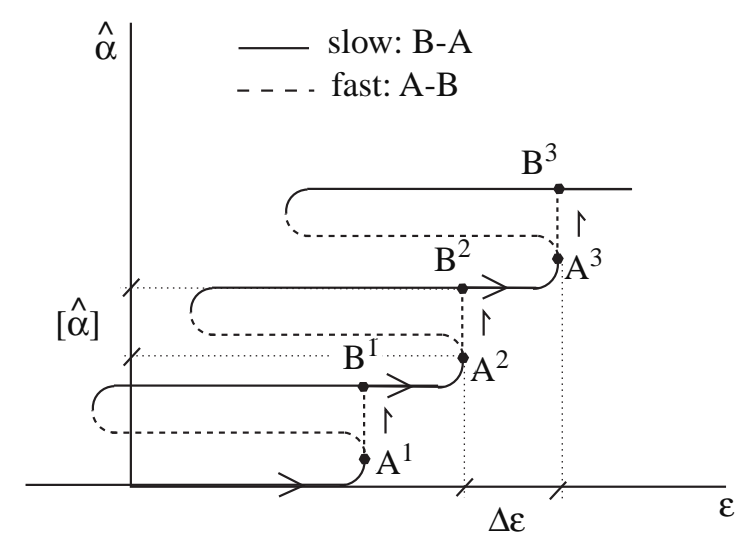

Fig. 1. Schematic representation of a multivalued equilibrium response $\alpha=\hat{\alpha}(\varepsilon)$ containing a sequence of turning points and a gradient flow-induced "maximum delay" trajectory exhibiting discontinuous slow-fast transitions (bold line). The system evolves through a succession of elastic steps $B^{i} \rightarrow A^{i+1}$, interrupted by fast plastic steps $A^{i} \rightarrow B^{i}$. 
delay" strategy when the system remains in a given metastable state until it becomes unstable.

During each slow-fast transition the dissipation is finite. Indeed, if we integrate the dissipation rate $D$ over the time required for the transition and neglect the variation of $\varepsilon$ we obtain $\int \mathrm{d} q^{\prime}=-\int(\partial f / \partial \alpha) \mathrm{d} \alpha=-[|\hat{f}|]$, where $[|\hat{f}|]$ is the discontinuity of the equilibrium free energy associated with a jump of $\hat{\alpha}$ at constant $\varepsilon$. On the contrary, the continuous evolution between two successive jumps can be considered non-dissipative and we can write $\int \mathrm{d} w=\int(\partial \hat{f} / \partial \varepsilon) \mathrm{d} \varepsilon=\Delta \hat{f}$, where $\Delta \hat{f}$ is the increment of the equilibrium free energy between the two jumps. In the presence of discontinuities the differential Gibbs relation (2.1) must be replaced by an integral relation

$$
\int \mathrm{d} w-\int \mathrm{d} \hat{f}=\int \mathrm{d} q^{\prime} \geqslant 0
$$

Since the free energy $\hat{f}$ is a discontinuous function of $\varepsilon$ its (distributional) derivative is a measure with a non-trivial singular part

$$
\int \mathrm{d} \hat{f}=\sum \int(\partial \hat{f} / \partial \varepsilon) \mathrm{d} \varepsilon+\sum[|\hat{f}|]
$$

Given that all dissipation is associated with discontinuities, we obtain $\int \mathrm{d} w=\sum \Delta \hat{f}$ (absolutely continuous part) and $\int \mathrm{d} q^{\prime}=-\sum[|\hat{f}|]$ (singular part); the situation here is reminiscent of nonlinear elasticity where in the inviscid limit dissipation takes place exclusively on shocks.

The total amount of dissipation depends on the density of jump points. Suppose that the jump point distribution is controlled by a small dimensionless parameter $\delta$; since the spacing is regular, we may put $\delta=\Delta \varepsilon$, where $\Delta \varepsilon$ is the strain increment associated with elastic deformation. We can then write $\sum_{i}^{n(t)}[|\hat{f}|]_{i}=n(t)[|\hat{f}|]$, where $n(t)$ is the number of jumps and $[|\hat{f}|]$ is the magnitude of the elementary energy discontinuity. In the limit $\delta \rightarrow 0$ we obtain $\mathrm{d} \varepsilon=\Delta \varepsilon \mathrm{d} n$ which gives

$$
D=-v \lim _{\delta \rightarrow 0} \frac{[|\hat{f}|]}{\Delta \varepsilon} .
$$

If the limit in Eq. (2.7) is finite we obtain the desired result that $D \sim v$. The only remaining step is to relate the rate of dissipation to the rate of change of the internal variable $\hat{\alpha}$. Suppose that the variation of $\hat{\alpha}$ outside the discontinuities is negligible and write $\hat{\alpha}(t)=\sum_{i}^{n}(t)[|\hat{\alpha}|]_{i}=n(t)[|\hat{\alpha}|]$, where $[|\hat{\alpha}|]$ is the elementary jump of $\hat{\alpha}$. Then

$$
\dot{\hat{\alpha}}=v \lim _{\delta \rightarrow 0} \frac{[|\hat{\alpha}|]}{\Delta \varepsilon}
$$

and if again the limit in Eq. (2.8) is finite, we obtain $\dot{\hat{\alpha}} \sim v$. The macroscopic dissipative potential along the loading path $(v>0)$ can then be written as

$$
D=g \dot{\hat{\alpha}}
$$


where the configurational force is

$$
g=-\lim _{\delta \rightarrow 0} \frac{[|\hat{f}|]}{[|\hat{\alpha}|]} .
$$

Since $g$ is assumed to be a constant, the resulting macroscopic dissipation is rate independent. Formula (2.10), replacing the classical $g=-\partial f / \partial \alpha$, is a consequence of the underlying assumptions concerning the similarity of the statistical distributions for $[|\hat{f}|]_{i}$ and $[|\hat{\alpha}|]_{i}$.

To establish the balance between the stored and the dissipated energies we need to compute the ratio $\beta=D / \dot{w}$. In the expression for the work one can neglect the contribution due to discontinuities and write $w(t)=\sum_{i}^{n} \Delta_{i} \hat{f}=n \Delta \hat{f}$, which gives in the continuum limit

$$
\beta=-\lim _{\delta \rightarrow 0} \frac{[|\hat{f}|]}{\Delta \hat{f}} .
$$

Under the assumption that the equilibrium locus $\hat{\alpha}(\varepsilon)$ is symmetric for loading and unloading, the analysis of the case $v<0$ (unloading) is straightforward.

\subsection{Yield stress}

The expected macroscopic behavior of the system in the continuum limit is illustrated in Fig. 2 which shows the equilibrium energy $\hat{f}(\varepsilon)$ and the associated stress-strain relation $\sigma(\varepsilon)$.

Observe that since the function $\hat{f}(\varepsilon)$ at finite $\delta$ is only piece-wise continuous, the equilibrium stress-strain relation $\hat{f}^{\prime}(\varepsilon)$, formally extended to discontinuities, will contain Dirac-function-type singularities located in the branch switching points. In the limit $\delta \rightarrow 0$ the singular part of the derivative $\hat{f}^{\prime}(\varepsilon)$ converges (weakly) to an absolutely continuous function $\lim _{\delta \rightarrow 0} \hat{f}^{\prime}(\varepsilon)$ which represents the equilibrium stress-strain relation (path $\mathrm{O}-\mathrm{P}-\mathrm{S}-\mathrm{T}$ ). The singular contributions to the equilibrium

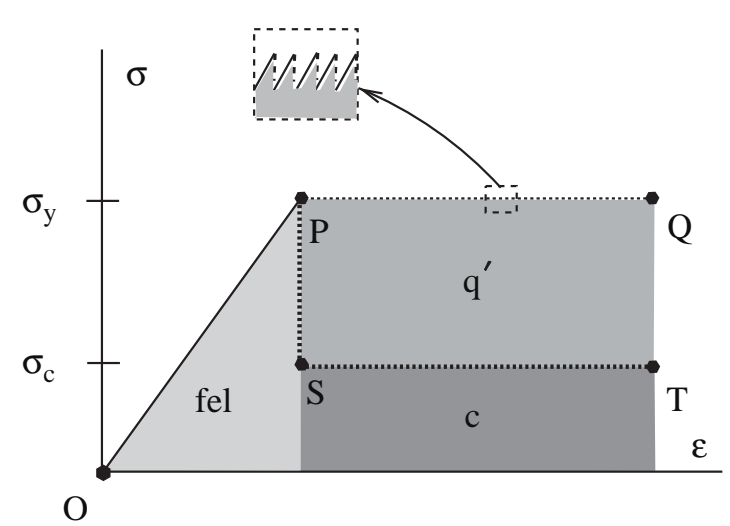

(a)

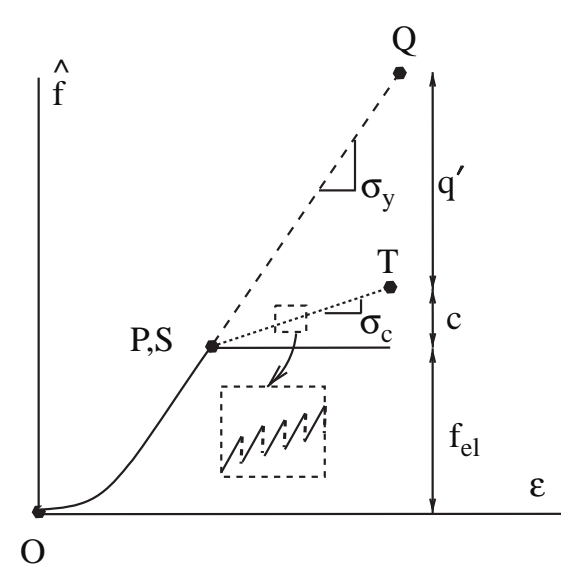

(b)

Fig. 2. Schematic representation of the effective stress-strain (a) and energy-strain (b) relations for a plastic body under quasi-static loading: O-P-S-T equilibrium response; $\mathrm{O}-\mathrm{P}-\mathrm{Q}$ actual response. 
stress-strain relation are probably not experimentally detectable due to viscous and inertial effects, so that the actual (measurable) stress-strain curve omits the precipitous dissipation and represents the limit of only the regular part of the energy function (path $\mathrm{O}-\mathrm{P}-\mathrm{Q}$ ).

To make the distinction between the actual and the equilibrium stress-strain relations quantitative, we notice that the actual yield stress $\sigma_{y}$ enters the expression for the increment of plastic work $\mathrm{d} w=\sigma_{y} \mathrm{~d} \varepsilon$, while the equilibrium yield stress $\sigma_{c}$ appears in the expression for the increment of the stored energy $\mathrm{d} c=\mathrm{d} w-\mathrm{d} q^{\prime}=$ $\sigma_{c} \mathrm{~d} \varepsilon$. Therefore in the continuum limit we obtain

$$
\sigma_{y}=\lim _{\delta \rightarrow 0} \frac{\Delta \hat{f}}{\Delta \varepsilon} \quad \text { and } \quad \sigma_{c}=\lim _{\delta \rightarrow 0} \frac{\Delta \hat{f}+[|\hat{f}|]}{\Delta \varepsilon} .
$$

Observe that $\sigma_{c}=(1-\beta) \sigma_{y}$, meaning that if $\beta=1$ all plastic work is fully dissipated making the equilibrium yield stress equal to zero (classical plasticity) while the actual yield stress $\sigma_{y}$ remains finite. If measurements recover even a portion of the singular contribution to the stress, one can expect a nucleation peak on the stress-strain curve. Although such a peak is indeed observed routinely in both metal and transformational plasticity, it is probably of a different physical origin (e.g. Truskinovsky and Vaichtein, 2004b).

\subsection{Zero-dimensional model}

To illustrate the general formulas obtained above we consider in the rest of this section a "toy" model which has an advantage of being fully analytical. We define the energy density as

$$
f(\varepsilon, \alpha)=\frac{E}{2}(\varepsilon-\alpha)^{2}+\sigma_{M} \alpha-k \delta \cos \left(\frac{\alpha}{\delta}\right) .
$$

The first term represents the elastic energy relative to the plastic reference strain $\alpha$. The second term is the energy of the reference state which is taken to be proportional to $\alpha$; the coefficient $\sigma_{M}$ is the analog of the Maxwell stress in the theory of phase transitions. The third term, mimicking a periodic pinning converges, in the continuum limit to zero (though not its first derivative). As in the previous section, we suppose that the total strain $\varepsilon$ is the controlling parameter and assume that the strain rate is constant and positive $v=\dot{\varepsilon}>0$ (loading). The relaxational dynamics associated with energy (2.13) is governed by the equation

$$
v \dot{\alpha}=E(\varepsilon-\alpha)-\sigma_{M}-k \sin \left(\frac{\alpha}{\delta}\right)
$$

where the derivative is taken with respect to the slow time $\tau$. Energy functions closely related to Eq. (2.13) have been employed in the modeling of a variety of phenomena from friction (Heslot et al., 1994) to plasticity (Abeyaratne et al., 1996; Choksi et al., 1999; Deseri and Owen, 2002; Bhattacharia, 1999). A new element in our analysis is the consideration of the concurrent limits $\delta \rightarrow 0$ and $v \rightarrow 0$ taken in both orders.

Following the general methodology presented in the beginning of this section we consider first, the limit $v \rightarrow 0$ and begin with identifying the jump points. The 
equilibrium locus can be found from the condition $\partial f / \partial \alpha=0$ which can be written as

$$
E(\varepsilon-\alpha)=\sigma_{M}+k \sin \left(\frac{\alpha}{\delta}\right) .
$$

It is natural to distinguish the case of weak pinning when $E>k / \delta$ and the function $\alpha=\hat{\alpha}(\varepsilon)$ solving Eq. (2.15) is monotone and the case of strong pinning $E<k / \delta$ when the system exhibits metastability and the function $\hat{\alpha}=\hat{\alpha}(\varepsilon)$ is multivalued. When the pinning is weak the viscous dynamics operates at both micro- and macro-levels and in the quasi-static limit the motion may be considered non-dissipative. On the contrary, in the case of strong pinning the macro and micro dissipative laws are different and the macro dynamics remains dissipative even in the quasi-static limit. Since our goal is the analysis of the case $\delta \rightarrow 0$, we can always assume that the condition of strong pinning is satisfied.

To find the turning points of the equilibrium response we need to solve simultaneously Eq. (2.15) and $\partial^{2} f / \partial \alpha^{2}=0$. We obtain the following parametric representation:

$$
\varepsilon=\alpha+\frac{\sigma_{M}}{E} \pm \frac{k}{E} \sqrt{1-\frac{E^{2} \delta^{2}}{k^{2}}}, \quad \sin \left(\frac{\alpha}{\delta}\right)= \pm \sqrt{1-\frac{E^{2} \delta^{2}}{k^{2}}} .
$$

The graphs of the equilibrium energy-strain $\hat{f}(\varepsilon)$ and the actual (measurable) stress-strain relation $\sigma(\varepsilon)$ are shown in Fig. 3. One can see that they reproduce the general structure of the graphs sketched in Fig. 2.

In the continuum limit $\delta \rightarrow 0$ the locus of the instability points (2.16) converges to the straight lines

$$
\alpha^{ \pm}(\varepsilon)=\varepsilon-\frac{\sigma_{M}}{E} \mp \frac{k}{E},
$$

with the two signs corresponding to loading and unloading, accordingly. Along the loading path $(v>0)$ we obtain the following asymptotic values of the relevant microparameters: $\Delta \hat{\alpha}=0,[|\hat{\alpha}|]=\Delta \varepsilon=2 \pi \delta, \Delta \hat{f}=2 \pi \delta\left(k+\sigma_{M}\right)$, and $[|\hat{f}|]=-2 \pi k \delta$. Then

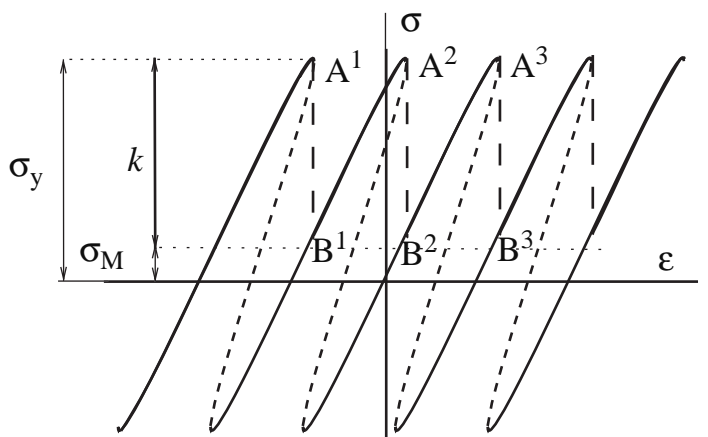

(a)

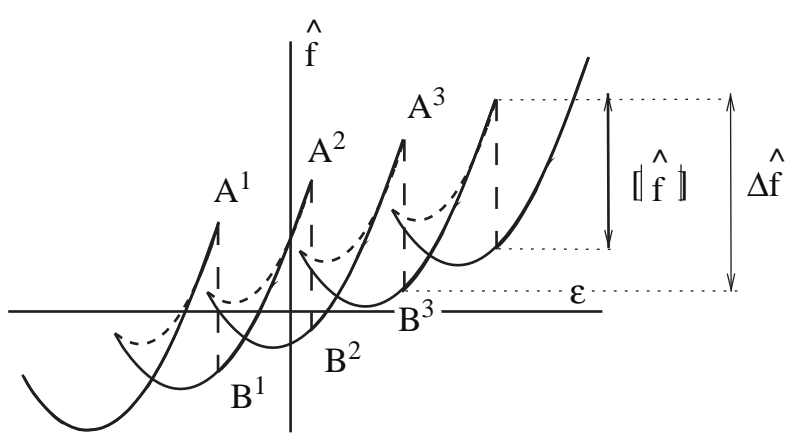

(b)

Fig. 3. Equilibrium stress-strain (a) and energy-strain (b) relations for model (2.13). Thin solid lines indicate metastable equilibria, thin dashed lines - unstable equilibria. The maximum delay trajectory is shown by bold lines. Parameters: $k=0.1, \delta=0.05, E=0.4, \sigma_{M}=0.02$. 
we can apply the formulas from the previous subsections to show that the viscous microscopic dynamics (2.14) generates at the macroscopic level rate-independent plastic behavior with a dissipative potential

$$
D=k \dot{\hat{\alpha}} \text {. }
$$

We also obtain automatically that the actual yield stress is $\sigma_{y}=\sigma_{M}+k$, the equilibrium yield stress is $\sigma_{c}=\sigma_{M}$, and that the heat to work ratio is $\beta=$ $k /\left(k+\sigma_{M}\right)$.

\subsection{Continuum dynamics}

Next we can reverse the order of the limiting procedures and consider the dynamics at $\delta=0$ and finite $v$. We first fix the loading parameter $\varepsilon$ and suppose that the micro-solution $\alpha(\tau ; \delta)$ of the equation (2.14) converges as $\delta \rightarrow 0$ to the macrosolution $\bar{\alpha}(\tau)$. One can show (Fisher, 1985; Risken, 1989; Abeyaratne et al., 1996) that for $\alpha^{-}<\bar{\alpha}<\alpha^{+}$the limiting system is trapped and $\dot{\bar{\alpha}}=0$. For $\bar{\alpha}>\alpha^{+}$(the case $\bar{\alpha}<\alpha^{-}$is symmetric) the dynamics is smooth but as the threshold $\bar{\alpha}=\alpha^{+}$is approached from above the motion becomes more and more jerky. Exactly at the threshold the convergence should be understood in the weak sense because $\dot{\bar{\alpha}}$ oscillates between 0 and $\infty$ and the motion is stick-slip. In the limit $\delta \rightarrow 0$ we can, following Abeyaratne et al. (1996), explicitly homogenize out small scales and write the macroscopic solution of Eq. (2.14) as a quadrature

$$
-v \int^{\bar{\alpha}(\tau)} \frac{1}{\pi} \int_{-1}^{1} \frac{\mathrm{d} s}{\left(-E(\varepsilon-\alpha)+\sigma_{M}+k s\right) \sqrt{1-s^{2}}} \mathrm{~d} \alpha=\tau .
$$

The integration in $s$ is explicit and for $\bar{\alpha}>\alpha^{+}$we obtain (Fisher, 1985; Risken, 1989; Abeyaratne et al., 1996)

$$
v \dot{\bar{\alpha}}=\sqrt{\left(E(\varepsilon-\bar{\alpha})-\sigma_{M}\right)^{2}-k^{2}} .
$$

This macroscopic kinetic law can be interpreted phenomenologically as the Bingham-type viscoelasticity with a threshold at $\bar{\alpha}=\alpha^{+}$(see Fig. 4). To obtain the rate-independent plasticity we need to consider an additional limit $v \rightarrow 0$. First notice that the threshold corresponds exactly to the yield stress $\sigma_{y}=k+\sigma_{M}$, so when $v=0$ the system is confined to the regime where $\alpha=\alpha^{+}$and $\sigma=\sigma_{y}$. One can see that due to the square root singularity of the homogenized dynamics (2.19), the limiting behavior of the system at $v \rightarrow 0$ takes place at constant yield stress $\sigma_{y}$ and is rate independent. If the loading is turned on and the total strain $\varepsilon$ is changing quasistatically, the threshold value $\alpha^{+}$evolves according to Eq. (2.17), while the stress remains fixed. The macro dynamics follows exactly the maximum delay strategy and appears as a succession of infinitesimal jump discontinuities.

The emerging picture agrees with what we have obtained previously by taking the limiting procedures in the reverse order. The advantage of first performing the spatial homogenization is that in this way we are not constrained by the automation model (emerging when we first consider the limit $v \rightarrow 0$ ) and are able to obtain a 


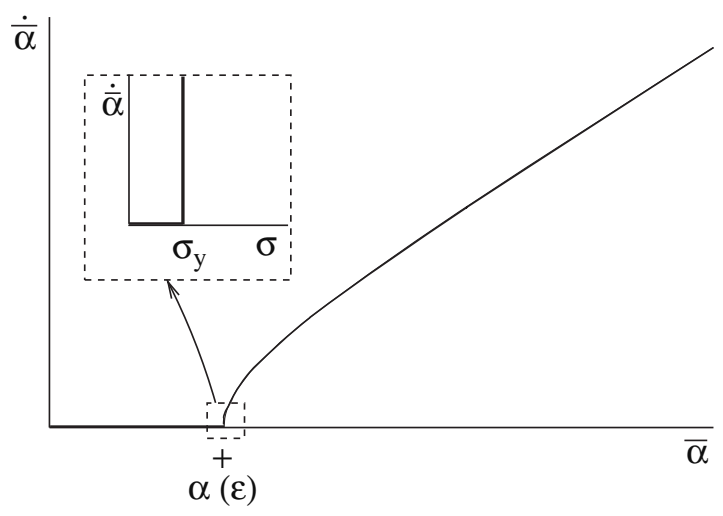

Fig. 4. Homogenized kinetics showing a rate-dependent viscoelastic response with a threshold. In the box we show schematically a rate-independent response at small strain rates around the threshold.

broader dynamical picture. The appearance of the square root singularity in the kinetic law explains why phenomenological modeling of rate-independent hysteresis requires power laws with singular exponents (Lubliner, 1998). In the framework of the "toy" model such rate-dependent viscoplastic regularization of the rateindependent plasticity can be obtained if the dynamical equation (2.14) is supplied with an additional source of white noise (Risken, 1989).

\section{One-dimensional model}

To obtain the simplest mechanical system generating an energy landscape of the desired complexity, consider $N$ identical elastic elements in series. By introducing discreteness, we assume that the system experiencing random pinning breaks into correlated volumes that behave coherently (Fisher, 1998). Denote the displacements of the nodes by $u_{i}$ and present the normalized elastic energy of the chain in the form

$$
f\left(\varepsilon_{1}, \ldots, \varepsilon_{N}\right)=N^{-1} \sum_{i=1}^{N} f_{0}\left(\varepsilon_{i}\right),
$$

where $\varepsilon_{i}=\left(u_{i+1}-u_{i}\right) / a$ are the strains, $a=L / N$ is the reference length, and $f_{0}\left(\varepsilon_{i}\right)$ is the normalized elastic energy of an element. The system is placed in a hard loading device with a prescribed average strain $\varepsilon$

$$
\varepsilon=N^{-1} \sum_{i=1}^{N} \varepsilon_{i}
$$

We suppose that the microscopic dynamics is governed by a gradient flow

$$
v \dot{\varepsilon_{i}}=-\frac{\partial \phi}{\partial \varepsilon_{i}}
$$


where

$$
\phi\left(\varepsilon_{1}, \ldots, \varepsilon_{N}\right)=N^{-1} \sum_{i=1}^{N}\left(f_{0}\left(\varepsilon_{i}\right)-\sigma \varepsilon_{i}\right)
$$

and $\sigma(\tau)$ is the total stress (Lagrange multiplier due to the constraint (3.2)). Referring to the discussion in the previous section, we can identify the vector $\varepsilon_{1}, \ldots, \varepsilon_{N}$ with the parameter $\alpha$ and assume that in quasi-static loading $\varepsilon(\tau)=\tau$. The two main dimensionless parameters of the problem are $v$ and $\delta=N^{-1}$.

To simulate plastic behavior we must assume that the elastic elements are (at least) bi-stable. This means that the energy function $f_{0}$ is non-convex with a double-well structure; for analytical simplicity we adopt the piece-wise parabolic approximation (see Fig. 5)

$$
f_{0}(\varepsilon):= \begin{cases}\frac{E_{0}}{2}\left(\varepsilon+\varepsilon_{0}\right)^{2}+\sigma_{M} \varepsilon, & \varepsilon<-t, \\ \frac{E_{0}}{2}\left(\frac{t-\varepsilon_{0}}{t} \varepsilon^{2}+\varepsilon_{0}\left(\varepsilon_{0}-t\right)\right)+\sigma_{M} \varepsilon, & -t \leqslant \varepsilon \leqslant t, \\ \frac{E_{0}}{2}\left(\varepsilon-\varepsilon_{0}\right)^{2}+\sigma_{M} \varepsilon, & t<\varepsilon .\end{cases}
$$

The two domains of convexity of $f_{0}$ (intervals $\varepsilon<t$ and $\varepsilon>t$ ) constitute stable energy wells which are separated by an unstable spinodal domain; $\sigma_{M}$ is the Maxwell stress and the equilibrium strains are placed at $\pm \varepsilon_{0}$. The strength of the pinning/jamming is expressed through the magnitude of the spinodal stress $\sigma_{s}=\sigma_{M}+E_{0}\left(\varepsilon_{0}-t\right)$. We assume that the condition of strong pinning $t<\left(1-N^{-1}\right) \varepsilon_{0}$ (Puglisi and Truskinovsky, 2000) is satisfied which is always appropriate in the continuum limit. In the case of transformational plasticity the convex energy wells can be directly associated with the stable phases of material.

Following the methodology presented in the previous section, we consider first the limit $v \rightarrow 0$ and begin with the reconstruction of the equilibrium locus. The strains inside the individual elements must satisfy the equilibrium equations $f_{0}^{\prime}\left(\varepsilon_{i}\right)=$ $\sigma, i=1, \ldots, N$. For a given $\sigma$, each equilibrium equation may have up to three

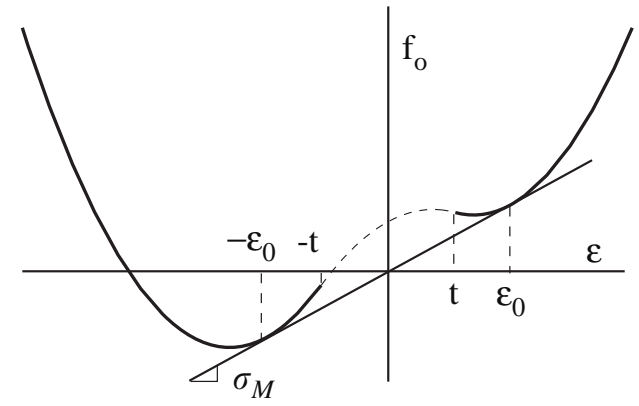

(a)

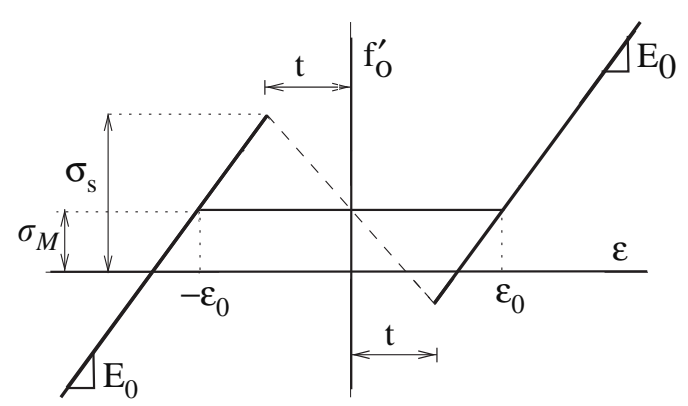

(b)

Fig. 5. Energy-strain (a) and stress-strain (b) relations for an individual bi-stable element. Here $\sigma_{M}$ is the Maxwell stress, $\sigma_{s}$ is the spinodal stress, $\pm \varepsilon_{0}$ are the equilibrium strains. 
different solutions

$$
\varepsilon_{I}=\frac{\sigma-\sigma_{M}}{E_{0}}-\varepsilon_{0}, \quad \varepsilon_{I I}=\frac{t}{t-\varepsilon_{0}} \frac{\sigma-\sigma_{M}}{E_{0}}, \quad \varepsilon_{I I I}=\frac{\sigma-\sigma_{M}}{E_{0}}+\varepsilon_{0} .
$$

Due to the invariance of the energy under permutations of the elements, the equilibrium state at a given $\varepsilon$ depends on the configuration only through the number of elements of type $I, I I$, and $I I I$ and we can parameterize the iso-energetic equilibrium branches by the phase fractions $p, q, r$, where $N p, N q, N r$ are the numbers of elements of type $I, I I$, and $I I I$, accordingly. If the phase composition is prescribed, the equilibrium stress-strain relation can be written as

$$
\sigma(\varepsilon)=\sigma_{M}+E\left(\varepsilon-\varepsilon_{p}\right)
$$

where

$$
E=\frac{\varepsilon_{0}-t}{\varepsilon_{0}-t-q \varepsilon_{0}} E_{0} \quad \text { and } \quad \varepsilon_{p}=(r-p) \varepsilon_{0} .
$$

It is natural to refer to $\varepsilon_{p}(\varepsilon)$ as plastic strain and to the remaining part $\varepsilon_{e l}=\varepsilon-\varepsilon_{p}$ as elastic strain; notice that the total stress $\sigma$ is a function of the elastic strain only. The normalized energy of the equilibrium configurations can be written as

$$
\hat{f}(\varepsilon)=\frac{1}{2} E\left(\varepsilon-\varepsilon_{p}\right)^{2}+\sigma_{M} \varepsilon+q \frac{1}{2} E_{0} \varepsilon_{0}\left(\varepsilon_{0}-t\right) .
$$

The stability properties of the equilibrium branches (3.7) were studied by Puglisi and Truskinovsky (2000) where it was shown that in the strong pinning regime all branches with $q \neq 0$ are unstable. The metastable branches are then parameterized only by the plastic strain; each branch is defined in its own strain domain bounded by $\varepsilon=\varepsilon_{p} \pm\left(\varepsilon_{0}-t\right)$. The energies of the metastable branches are represented by displaced parabolas

$$
\hat{f}(\varepsilon)=\frac{1}{2} E_{0}\left(\varepsilon-\varepsilon_{p}\right)^{2}+\sigma_{M} \varepsilon
$$

Observe that at each value of $\varepsilon$, we obtain a finite number of local minima of the energy. They are parameterized by $\varepsilon_{p}(\varepsilon)$ which suggests the identification $\hat{\alpha}(\varepsilon)=\varepsilon_{p}$. Since the so defined variable $\hat{\alpha}$ takes only discrete values, the quasi-static dynamics also becomes discrete: the corresponding automation model circumvents the fast motions and represents the piece-wise adiabatic process as a succession of integer steps. Since the branch switching takes place when the current branch terminates we obtain again the maximum delay strategy.

Which particular stable state is selected when the system reaches the point of instability cannot be determined from the analysis of equilibria only and requires the reconstruction of the full non-equilibrium energy landscape. We shall discuss this question in the next subsection while here, anticipating the result, we simply postulate that the switching always takes place between branches which differ by the phase state of only one element. The complete transition from one homogeneous branch to the other can then be represented as a sequential combination of $N$ identical transitions inside individual elements. 


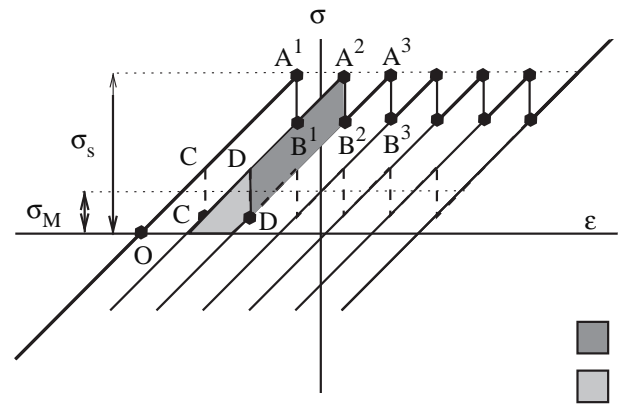

(a)

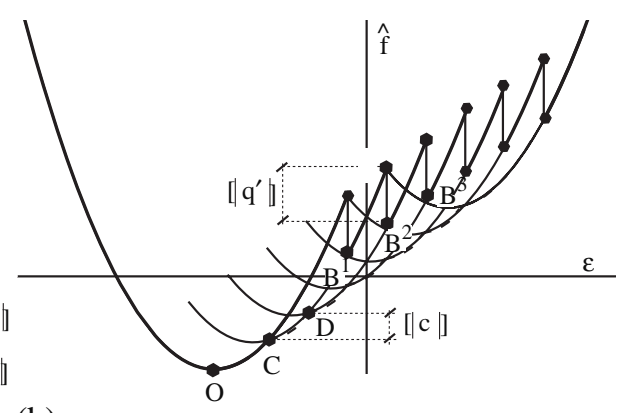

(b)

Fig. 6. Stress-strain (a) and energy-strain (b) relations for a chain with $N=6$; metastable locus - solid line, maximum delay path - bold line, global minimum - dashed line. Also shown are the incremental stored energy $[|c|]=\Delta f+[|f|]$ and the dissipated energy $[|q|]=-[|\hat{f}|]$ for a single step. The parameters are: $E_{0}=1, t=0.15, \varepsilon_{0}=1, \sigma_{M}=0.3$.

In the stress-strain space the maximum delay path is represented by a sawtooth curve (see Fig. 6). Each incremental step consists of an elastic stage $\left(B^{i} \rightarrow A^{i+1}\right)$ when the system follows a metastable branch and a plastic stage $\left(A^{i} \rightarrow B^{i}\right)$ when the system switches at fixed strain between two neighboring local minima. A computation shows that during each elastic step $\Delta \hat{\alpha}=0$ and $\Delta \varepsilon=2 \varepsilon_{0} / N$, while during each plastic step $[|\hat{\alpha}|]=2 \varepsilon_{0} / N,[|\varepsilon|]=0$; observe that the plastic strain increases exactly by the amount needed to relax the elastic strain. The stress drop associated with each plastic step is equal to $[|\sigma|]=-2 E_{0} \varepsilon_{0} / N$.

Next we analyze the energetics of the elastic and plastic steps. During an elastic step the external work transforms into elastic energy and

$$
\Delta \hat{f}=\frac{2 \varepsilon_{0}}{N}\left(\sigma_{s}-E_{0} \frac{\varepsilon_{0}}{N}\right) .
$$

During each plastic step the dissipation is equal to

$$
-[|\hat{f}|]=\frac{2}{N} \varepsilon_{0}\left(\sigma_{s}-\sigma_{M}-E_{0} \frac{\varepsilon_{0}}{N}\right) .
$$

After the completion of each incremental step, starting and ending at the same value of the (spinodal) stress $\sigma_{s}$, the elastic energy of the elements that do not change phase remains the same. The elastic energy of the element that does change phase increases by the amount $\Delta \hat{f}+[|\hat{f}|]=2 \varepsilon_{0} \sigma_{M} / N$ which is accumulated by the body as stored energy. In the continuum limit $\delta \rightarrow 0$, the sawtooth path on the stress-strain plane converges to a horizontal plateau at $\sigma_{y}=\sigma_{s}$, representing the "actual" stress while the limiting value of the "equilibrium" stress is equal to the Maxwell stress $\sigma_{c}=\sigma_{M}$. The configurational force converges to $g=\sigma_{s}-\sigma_{M}$, and the fraction of the dissipated energy in the continuum limit is equal to $\beta=\left(\sigma_{s}-\sigma_{M}\right) / \sigma_{s}$. Due to the symmetry of the stress-strain diagram, the loading and unloading paths are symmetric. Over the complete hysteresis cycle the number of individual dissipative events is equal to $2 N$ which gives for the total dissipation $2 N[|\hat{f}|]=-4 \varepsilon_{0}\left(\sigma_{s}-\sigma_{M}-\right.$ $\left.E_{0} \varepsilon_{0} / N\right)$. The total plastic strain is equal to zero and the stored energy accumulated 
during the direct path is fully returned on the reverse path. In the continuum limit the total dissipation in a cycle is equal to the area inside the hysteresis loop $\oint D \mathrm{~d} t=$ $4 \varepsilon_{0}\left(\sigma_{s}-\sigma_{M}\right)$.

\subsection{Energy landscape}

To understand the branch selection process along the path of maximal delay it is necessary to reconstruct the non-equilibrium energy landscape $f(\varepsilon, \alpha)$. We begin with a simple example of a system with $N=3$, assuming that the strong pinning condition $t<2 \varepsilon_{0} / 3$ is satisfied.

A generic configuration of a chain with three elements placed in a hard device can be described by two coordinates: symmetry considerations (Puglisi and Truskinovsky, 2000) suggest the use of the barycentric-type variables $\xi$ and $\psi$ defined as $\varepsilon_{1}=$ $\varepsilon+\xi+\sqrt{3} \psi, \varepsilon_{2}=\varepsilon+\xi-\sqrt{3} \psi$, and $\varepsilon_{3}=\varepsilon-2 \xi$. In terms of the new variables the energy of the system can be written as

$$
f=\frac{1}{3}\left(f_{0}(\varepsilon+\xi+\sqrt{3} \psi)+f_{0}(\varepsilon+\xi-\sqrt{3} \psi)+f_{0}(\varepsilon-2 \xi)\right) .
$$

To visualize this energy function we choose four particular values of the average strain $\varepsilon$, specified on the equilibrium stress-strain and energy-strain diagrams in Fig. 7. The corresponding energy landscapes are presented in Fig. 8.

At $\varepsilon \ll \varepsilon_{a}$ (see Fig. 7) the only equilibrium configuration is the minimum $m_{1}$ representing the trivial branch $(3,0,0)$, where in parentheses we indicate the phase configuration $(p N, q N, r N)$. At $\varepsilon=\varepsilon_{a}$ we see an additional set of non-trivial local minima $m_{2}$, corresponding to the two-phase configurations $(2,0,1)$. According to Fig. 8a,b the minimal barrier paths leading away from $m_{1}$ go trough saddles $s_{1}$, (configurations $(2,1,0)$ with one element in the spinodal region). These paths are represented in Fig. 8a,b by the straight lines $\psi=0\left(\varepsilon_{1}=\varepsilon_{2}\right), \psi=\sqrt{3} \xi\left(\varepsilon_{2}=\varepsilon_{3}\right)$, and

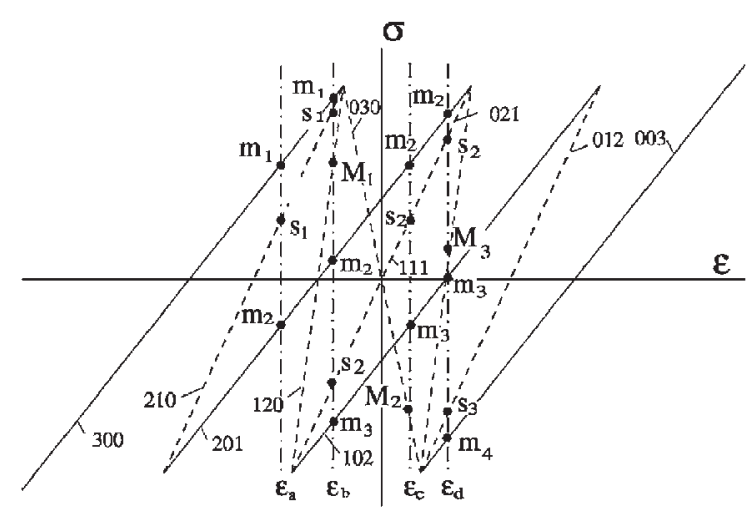

(a)

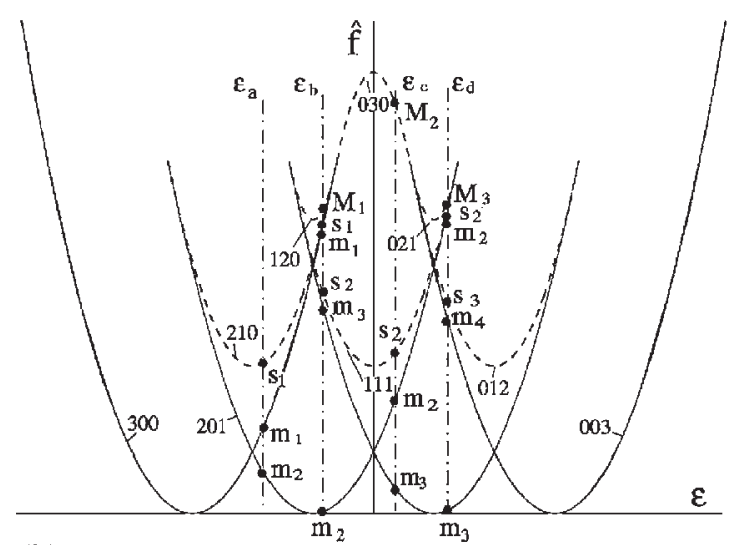

(b)

Fig. 7. Equilibrium energy-strain (a) and stress-strain (b) diagrams for a bi-stable chain with $N=3$. The numbers in parentheses give the phase configuration $(p N, q N, r N)$. Solid lines indicate metastable states (local minima $m_{i}$ ), dashed lines - unstable states (saddle points $s_{i}$ and local maxima $M_{i}$ ). Parameters: $t=0.2, E_{0}=1, \varepsilon_{0}=1$, and $\sigma_{M}=0$. The energy landscapes at the four indicated values of $\varepsilon$ are shown in Fig. 8 . 

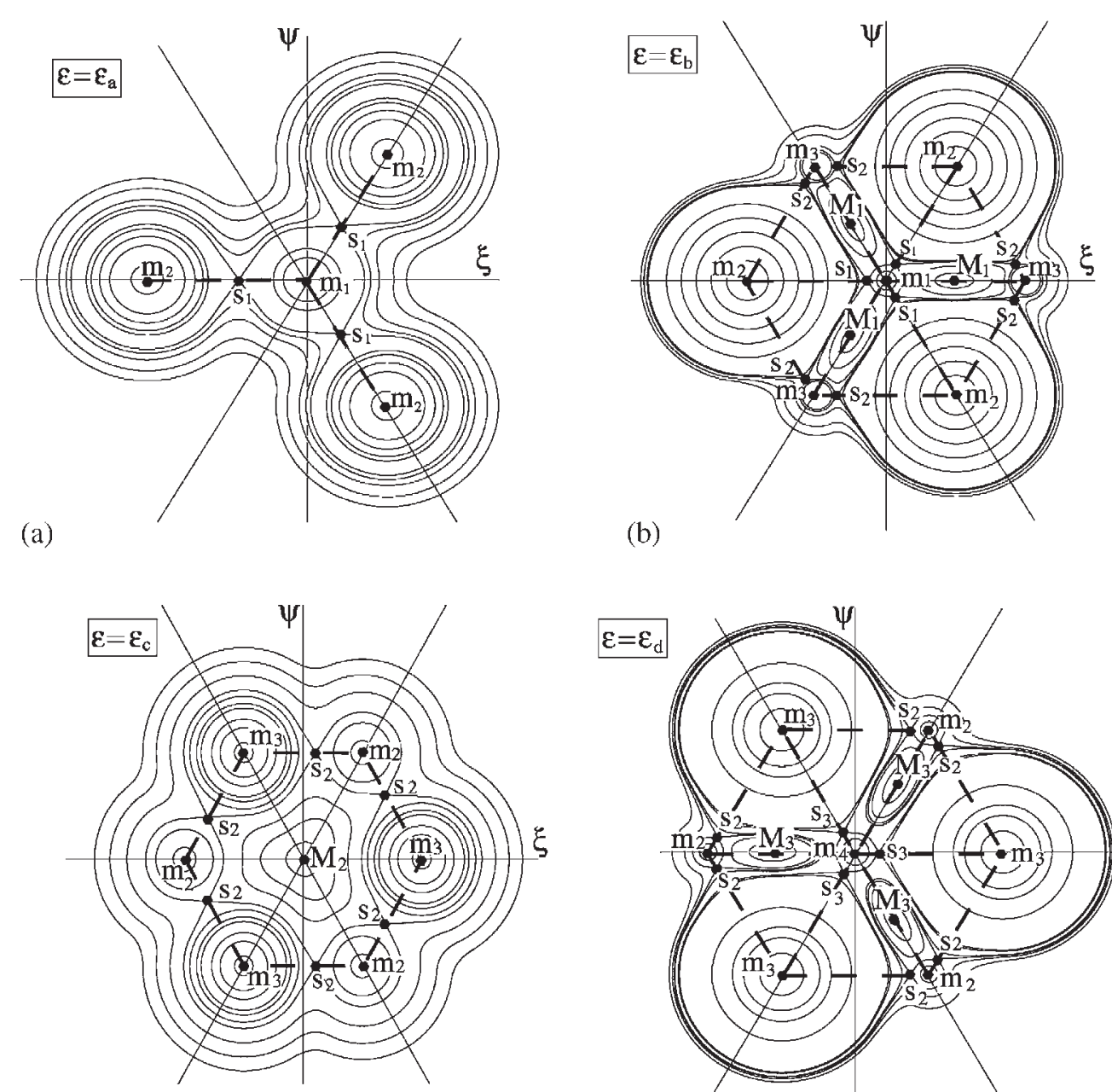

(c)

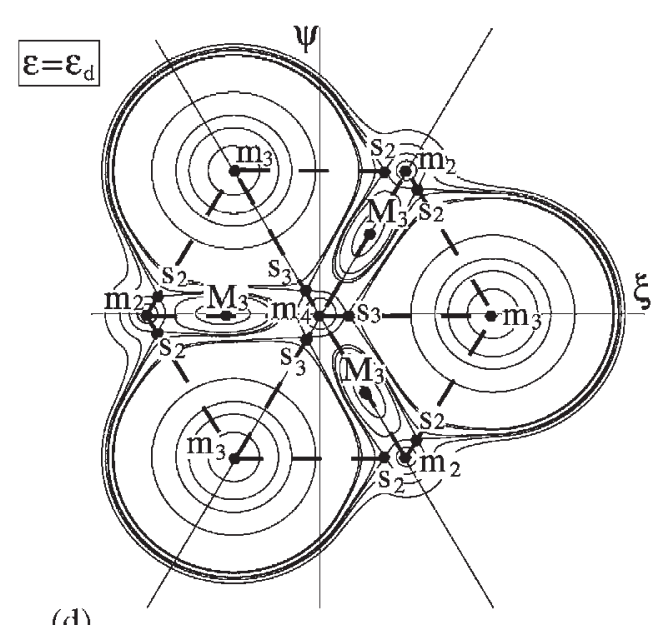

Fig. 8. (a-d) Energy landscapes for a bi-stable chain with three elements $(N=3)$ at the four values of the total strain $\varepsilon$ indicated in Fig. 7. Local minima are denoted by $m_{i}$, saddle points by $s_{i}$, local maxima by $M_{i}$. The dashed lines are minimum energy barrier paths. Parameters: $t=0.2, E_{0}=1, \varepsilon_{0}=1$, and $\sigma_{M}=0$.

$\psi=-\sqrt{3} \xi\left(\varepsilon_{1}=\varepsilon_{3}\right)$. Along the corresponding trajectories two elements out of three remain mechanically equilibrated and stay in the same energy well, while the third one changes phase. The extensions of these trajectories beyond the saddles $s_{1}$ provide the paths of steepest descent to the local minima $m_{2}$.

As the average strain $\varepsilon$ increases the saddles $s_{1}$ collapse on the local minimum $m_{1}$ making it unstable. Implying the presence of fluctuations, one can argue that at this bifurcation point the paths $m_{1}-s_{1}-m_{2}$ are preferred as securing the minimal barriers already before the bifurcation point is reached. Therefore we postulate that the branch switching event is the transition $m_{1}-m_{2}$.

After the transition the system remains in the two-phase configuration $m_{2}$ until the corresponding local minimum ceases to exist. According to Fig. 8b,c,d the minima $m_{2}$ and $m_{3}$ are connected by the straight trajectories $\psi= \pm \sqrt{3} \xi \mp(2 / \sqrt{3}), \psi=$ $\mp \sqrt{3} \xi \mp(2 / \sqrt{3})$, and $\psi= \pm(2 / 3)$, passing through the saddles $s_{2}$. Along these paths two elements remain mechanically equilibrated while the third one changes phase. These trajectories provide the minimum barrier paths leading away from the basin of $m_{2}$; they are also the paths of steepest descent from the saddles $s_{2}$ to the minima $m_{3}$. 
At the next bifurcation point the saddles $s_{2}$ merge with the local minima $m_{2}$ making them unstable and we can again argue that the paths $m_{2}-s_{2}-m_{3}$ are preferred meaning that the system will undergo the transition $m_{2}-m_{3}$. Finally, the minimal barrier trajectories leading away from the local minima $m_{3}$ pass through the saddles $s_{3}$ as the last element changes its phase; their extension links these saddles to the trivial minimum $m_{4}$.

The above analysis suggests that along the minimal barrier trajectory the incremental plastic deformation is always localized in a single element and that the elements transform in sequence, one after another. This means that at each threshold there is one slow manifold (up to the symmetries) which dominates the motion and that the system behaves as a "toy" model inside the effective elastic potential of the other elements (generalized Peierls-Nabarro (PN) potential). In the automation model presented in the previous section the internal variable took only discrete values representing the positions of the minima of the PN potential and the continuous dynamics was replaced by a map between the metastable configurations.

To reconstruct the PN potential for the case of arbitrary $N$, consider a generic local minimum $A$, characterized by the phase fractions $r N=\hat{k}, p N=0, q N=$ $N-\hat{k}$. Due to the symmetry of the energy we can always assume that the first $\hat{k}$ elements are in the first energy well while the rest are in the second energy well. Following the above example, consider the path connecting $A$ with another local minimum $B$ of the type $(\hat{k}-1,0, N-\hat{k}+1)$ and passing through the saddle $S$ of the type $(\hat{k}-1,1, N-\hat{k})$. By using the stress $\tau$ in the non-transforming springs as a parameter we can write an explicit representation for the elemental strains along this trajectory

$$
\varepsilon_{i}(\tau)= \begin{cases}\frac{\tau-\sigma_{M}}{E_{0}}-\varepsilon_{0}, & i=1, \ldots, \hat{k}-1, \\ \hat{\varepsilon}(\tau, \varepsilon), & i=\hat{k}, \\ \frac{\tau-\sigma_{M}}{E_{0}}+\varepsilon_{0}, & i=\hat{k}+1, \ldots, N .\end{cases}
$$

The strain in the transforming element $\hat{\varepsilon}(\tau, \varepsilon)$ can be found from the constraint

$$
N \varepsilon=(\hat{k}-1)\left(\frac{\tau-\sigma_{M}}{E_{0}}-\varepsilon_{0}\right)+\hat{\varepsilon}(\tau, \varepsilon)+(N-\hat{k})\left(\frac{\tau-\sigma_{M}}{E_{0}}+\varepsilon_{0}\right) .
$$

The proof of the fact that the saddle $S$ indeed corresponds to the minimal barrier outside the basin of attraction of the local minimum $A$ and that path (3.11) is the trajectory of steepest descent from the saddle $S$ to the new metastable state $B$ can be reconstructed from the similar proof in the case of soft device (Puglisi and Truskinovsky, 2002b).

To define the collective coordinate which links different segments of the PN landscape and which can be identified with the global internal variable $\alpha$, we write

$$
\alpha(\tau, \varepsilon)=\varepsilon-\frac{\tau-\sigma_{M}}{E_{0}} .
$$


The advantage of this new parameterization is that $\alpha$ increases monotonically from $A$ to $B$ and is equal to $\varepsilon_{p}(\varepsilon)$ (plastic strain) in both metastable configurations. In terms of $\alpha$ the energy variation along trajectory (3.11) can be written in the form

$$
\begin{aligned}
f(\alpha, \varepsilon)= & \frac{1}{N}\left((\hat{k}-1) f_{0}\left(\frac{\tau(\alpha, \varepsilon)-\sigma_{M}}{E_{0}}-\varepsilon_{0}\right)\right. \\
& +f_{0}(\hat{\varepsilon}(\tau(\alpha, \varepsilon), \varepsilon))+(N-\hat{k}) f_{0}\left(\frac{\tau(\alpha, \varepsilon)-\sigma_{M}}{E_{0}}+\varepsilon_{0}\right) .
\end{aligned}
$$

Here $\tau(\alpha, \varepsilon)$ is the inversion of Eq. (3.13). One can see that $\partial f(\alpha, \varepsilon) / \partial \alpha=(1-$ $\left.N^{-1}\right)\left(f_{0}^{\prime}(\hat{\varepsilon})-\tau(\alpha, \varepsilon)\right)$, which confirms that the equilibria of the original energy are the critical points of $f(\alpha, \varepsilon)$ at constant $\varepsilon$. Along the segment of the trajectory from $A$ to $S$ the stress $f_{0}^{\prime}(\hat{\varepsilon})$ in the transforming element $\hat{k}$ is larger then the stress $\tau$ in the equilibrated elements giving $\partial f(\alpha) / \partial \alpha>0$; similarly, along the path going from $S$ to $B$, we obtain $\partial f(\alpha) / \partial \alpha<0$. The path $A-S-B$ provides a generic switching mechanism and by linking the successive $A-S-B$ segments one can reconstruct the complete PN potential. An example for a chain with $N=7$ is presented in Fig. 9 .

In addition to providing the energies of the metastable equilibria $m_{i}$ (analogs of points $A$ and $B$ above) the PN potential contains information about the minimal energy barriers $s_{i}$ separating these metastable equilibria (analogs of points $S$ ). This information is important if one considers the possibility of fluctuation-induced barrier crossing and, for instance, replaces the maximal delay strategy by an alternative strategy allowing the escape from a local minimum when the smallest barrier in the corresponding basin of attraction reaches below a given threshold $h$. To investigate this possibility we can express the normalized height of the minimal barrier for each individual transition $h=(f(S)-f(A)) / a$ as a function of the stress $\sigma$

$$
h(\sigma)=\frac{E_{0} \varepsilon_{0}}{2 L} \frac{\left(\left(\sigma-\sigma_{M}\right) / E_{0}+t-\varepsilon_{0}\right)^{2}}{\left(\varepsilon_{0}-\varepsilon_{0} / N-t\right)} .
$$

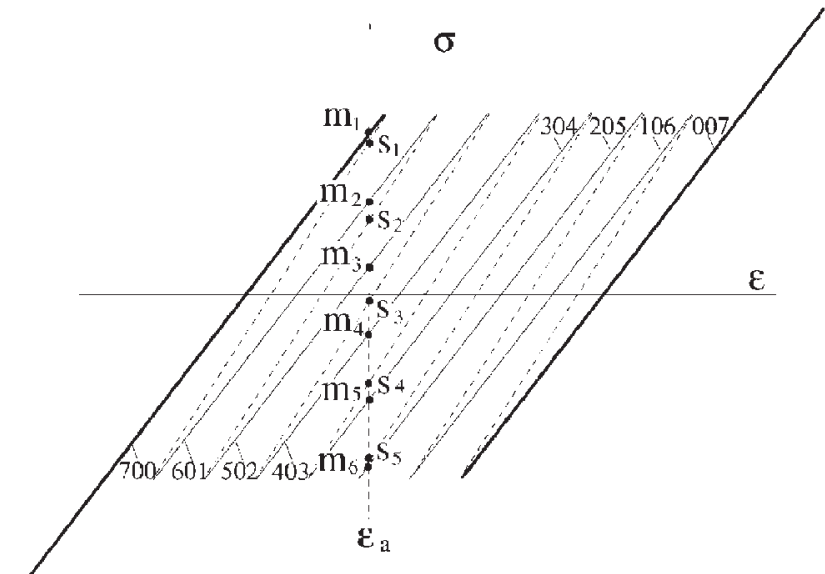

(a)

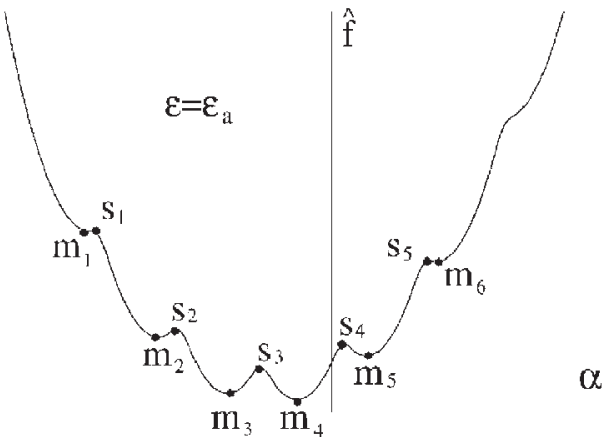

(b)

Fig. 9. Peierls-Nabarro (PN) energy landscape at a fixed total strain for a chain with $N=7$ (b); the relevant section of the equilibrium stress-strain diagram is shown in (a). Parameters: $t=0.2, E_{0}=1$, $\varepsilon_{0}=1$, and $\sigma_{M}=0$. 
Observe that if $h$ is fixed the transformation governed by this new strategy takes place at a constant stress which is independent of the current phase configuration and is constant during the complete transition from one trivial branch $(N, 0,0)$ to the other $(0,0, N)$. The barrierless yielding (maximum delay strategy) discussed in the previous section corresponds to the case $h=0$ and takes place at the spinodal stress $\sigma_{s}$.

If the possibility of both forward and reverse transitions is taken into consideration, the total rate of transformation becomes a function of the configurational force

$$
g(\sigma)=\frac{h_{A \rightarrow S}-h_{B \rightarrow S}}{a[|\hat{\varepsilon}|]}=\frac{N\left(\sigma-\sigma_{M}\right)-E_{0} \varepsilon_{0}}{N-1} .
$$

The configurational force is equal to zero at $\sigma=\sigma_{M}+E_{0} \varepsilon_{0} / N$ (discrete analogs of the Maxwell stress) where the barriers are the highest and takes the largest value at the spinodal stress $\sigma_{s}$ where the barriers are absent. This shows that the "minimal barrier' criterion is equivalent to the condition of a "maximal driving force". The driving force for the simultaneous transition of $n$ elements is a decreasing function of $n$, which means that any given "friction threshold" $g$ will first be reached for the transition with $n=1$ and that in the model with a prescribed threshold (see, for instance, Mielke et al. (2002)) the elements will be switching one at a time at a fixed transition stress.

In the continuum limit $\delta \rightarrow 0$ (or $N \rightarrow \infty$ ) the absolute size of the energy barriers decreases to zero while the configurational force remain finite. If the normalized barrier size $h$ is prescribed, the actual yield stress in the limiting problem can be found from

$$
\sigma_{y}=\sigma_{M}+E_{0}\left(\varepsilon_{0}-t-\sqrt{\frac{2 L h\left(\varepsilon_{0}-t\right)}{E_{0} \varepsilon_{0}}}\right) .
$$

Alternatively, in terms of the critical configurational force $g$ the actual yield stress can be expressed as

$$
\sigma_{y}=\sigma_{M}+g .
$$

\subsection{Continuum dynamics}

The next problem is to revert the order of the limiting procedures and to reconstruct the dynamics of the system at $\delta=0$ and finite $v$. Here we cannot simply reproduce the methodology used in the "toy" model because the limiting problem is infinite dimensional and the PDE analog of (2.19) is not straightforward.

The general character of the dynamics for a chain with bi-stable elements at finite $\delta$ can be seen from the analysis of the travelling waves in the discrete Frenkel-Kontorova (FK) model which becomes equivalent to Eq. (3.3) in the overdamped limit if linear next to nearest neighbor (NNN) interactions are taken into consideration (Truskinovsky and Vaichtein, 2003). The kinetic relation for the 
FK model at small average velocities (around the depinning threshold) is similar to what we obtained for the "toy" model exhibiting the same characteristic square root singularity. The motion is continuous at large velocities but becomes stick slip around the depinning threshold with periods of almost static evolution interrupted by sudden discontinuous advances of the transformation front (Carpio and Bonilla, 2003). The main deficiency of the model (J.J), which can be fixed by adding NNN interactions, is its permutational degeneracy in statics leading to nonphysical effects in dynamics (Truskinovsky and Vaichtein, 2004a, b).

To illustrate the complexity of the limiting problem, consider the structure of the metastable configurations at $\delta \rightarrow 0$. If we formally replace in the energy the finite differences by derivatives and sums by integrals, we obtain the problem: find $\inf _{u \in \mathscr{A}} \int_{0}^{L} f_{0}\left(u_{x}\right)$, where $\mathscr{A}:=\left\{u \in W^{1, p}((0, L)): \int_{0}^{L} u_{x}(x) \mathrm{d} x=\mathrm{d}\right\}$. The local minima in this continuum setting satisfy the Euler-Lagrange equation $f_{0}^{\prime}\left(u_{x}(x)\right)=\sigma$, whose weak solutions are exactly (3.6). For the given phase configuration the stress and the energy take the form (3.7), (3.9), provided that we define the phase fractions $p, q, r$ as measures, for instance $p L:=\mu\left\{x \in(0, L): u_{x}(x)<-t\right\}$. Also, exactly as in the discrete case, the Legendre-Hadamard stability condition $f_{0}^{\prime \prime}\left(u^{\prime}(x)\right)>0$ restricts the set of metastable phase configurations to the ones with $q=0$; the energy for the metastable configurations can then be read from Eq. (3.10). A peculiarity of the infinite-dimensional case is that depending on the topology, the meaning of the local minima may be different. Thus, taking the standard integral norm $\|u\|_{W^{1, p}}$ with $p \leqslant \infty$ we obtain that the Weierstrass necessary condition excludes all local minima that are not also the global ones (Ericksen, 1975). Exactly the same local minimizers as in our discrete problem with $N=\infty$ are recovered if, instead, we choose the norm $\|u\|_{W^{1, \infty}}$. Most of the recovered local minima, however, do not belong to a potential well (Ball et al., 1978). This makes the energy landscape "flat" in the sense that it is possible to escape from the basin of attraction of each of these metastable states without overcoming an energy barrier. To deal with this situation the phenomenological continuum models have been proposed by Faciu and Suliciu (1994) and Vainchtein and Rosakis (1999); while they were shown numerically to exhibit rate-independent hysteresis (with discretizationinduced serrations), their formal relation to the discrete model (3.3) remains to be established.

\section{Non-isothermal case}

In the previous sections we considered only isothermal systems. This was appropriate because the rate-independent dissipation is in many respects an athermal phenomenon for which non-trivial information can be already obtained from a purely mechanical description. The isothermal setting allowed us to avoid the explicit introduction of the entropy function for the plastically deformed body. To understand the associated difficulties we can consider a spatially homogeneous, nonisothermal system. To capture the discontinuities, we write the first and second laws 
of thermodynamics in the integral form

$$
\int \mathrm{d} e=\int \mathrm{d} w+\int \mathrm{d} q, \quad \int \theta \mathrm{d} \eta=\int \mathrm{d} q+\int \mathrm{d} q^{\prime} .
$$

Here $\theta$ is the temperature, $e(\varepsilon, \alpha, \theta)$ is the internal energy density $\eta(\varepsilon, \alpha, \theta)$ is the entropy density, and $\dot{q}$ is the rate of external heat supply. By adiabatically eliminating the internal variable $\alpha$ and taking into consideration singular components of the derivative of the internal energy we can write

$$
\int \mathrm{d} \hat{e}=\sum \int \frac{\partial \hat{e}}{\partial \varepsilon} \mathrm{d} \varepsilon+\sum \int \frac{\partial \hat{e}}{\partial \theta} \mathrm{d} \theta+\sum[|\hat{e}|] .
$$

If we additionally assume that the jump discontinuities are isothermal, we obtain the following equation for the entropy:

$$
\sum \int \theta \frac{\partial \hat{\eta}}{\partial \varepsilon} \mathrm{d} \varepsilon+\sum \int \theta \frac{\partial \hat{\eta}}{\partial \theta} \mathrm{d} \theta+\sum \theta[|\hat{\eta}|]=\int \mathrm{d} q+\int \mathrm{d} q^{\prime}
$$

where the dissipative term remains the same as before

$$
\int \mathrm{d} q^{\prime}=-\sum([|\hat{e}|]-\theta[|\hat{\eta}|])=-\sum[|\hat{f}|] .
$$

In the continuum limit we can localize (4.3) and obtain an equation for the temperature

$$
c \dot{\theta}+\theta\left(\frac{\partial \hat{\eta}}{\partial \varepsilon}+\lim _{\delta \rightarrow 0} \frac{[|\hat{\eta}|]}{\Delta \varepsilon}\right) \dot{\varepsilon}=r(\theta)-\lim _{\delta \rightarrow 0}\left(\frac{[|\hat{f}|]}{\Delta \varepsilon}\right) \dot{\varepsilon} .
$$

Here $c=\theta \partial \hat{\eta} / \partial \theta$ is the specific heat and $r(\theta)$ is the rate of convection or radiation; heat conduction can be added in the standard way for spatially inhomogeneous systems. The last term on the right-hand side of (4.5) is due to plastic dissipation; it has been discussed in detail in the previous sections. The two terms in parentheses on the left-hand side represent reversible elastic heating. The first term comes from the classical thermo-elasticity and is related to thermal expansion (Rosakis et al., 2000). The second term corresponds to the latent heat due to the discontinuous variation of the internal variable $\hat{\alpha}$. In order to compute the associated entropy discontinuity we need to assume that a defect can move from one pinned configuration to another through a sequence of constrained equilibrium states (Rice, 1970).

The purely mechanical model relies significantly on the presence of metastable configurations which is relevant only when elementary units have barriers to flipping large enough that thermal activation can be ignored. Therefore, our system does not come to thermal equilibrium even in the quasi-static limit meaning that the temperature $\theta$ is essentially equal to zero. If $\theta \neq 0$ then in addition to the two characteristic times: $t$-associated with gradient flow dynamics and $\tau$-associated with external loading, we have a new time scale $\bar{t}$-associated with the thermally activated relaxation involving a possibility of barrier crossing. For instance, in a system with a representative barrier size $h$, we have $1 / \bar{t} \sim \exp (-h /(k \theta))$ and $\bar{t}=\infty$ only if $\theta=0$. The quasi-static mechanical model discussed in the previous sections is 
appropriate in the case when $\bar{t} \gg \tau \gg t$. If, on the contrary, $\tau \gg \bar{t}$ and the loading is much slower than thermal relaxation, the maximum delay strategy will be replaced with the global energy minimization.

Here it is also appropriate to address the issue of cold work. It is known that in metal plasticity $\beta \sim 0.8-0.9$ because of the accumulation of metastable defects. The associated energy decays by thermal activation (Bever et al., 1972), which makes it different from our stored energy of completely equilibrium nature. Such equilibrium stored energy is relevant, for instance, in transformational plasticity where even in the absence of cold work $\beta \leqslant 1$ due to the difference in chemical energies of the phases. The equilibrium stored energy can also be linked to the energy of the slipinduced steps on the surface of plastic crystals that cannot be removed by annealing. The incorporation of the non-equilibrium energy of cold work into our model would require introduction of at least one additional internal variable with its own relaxational kinetics (Rosakis et al., 2000). By neglecting this phenomenon we tacitly assume that the rate of loading is slower than this relaxational process while it is still sufficiently fast to make the internal metastability possible.

\section{Conclusions}

The main objective of this paper was to demonstrate that classical plasticity can be in principle derived from a model with viscous dissipation as a result of a judicial elimination of small spatial scales and fast times. The singular dissipative structure emerges in this approach as a consequence of time averaging and spatial homogenization in a system with regular dissipative behavior.

The ingredient making such a reduction possible is the micro-metastability due to the presence of random imperfections. While these imperfections are small and hardly affect the mechanical properties of the crystal, they can change qualitatively the properties of the defect dynamics making breakaway from barriers rather than the force velocity relation for isolated defects a rate controlling mechanism; a quasistatic inelastic deformation may then be characterized as "punctuated equilibrium" (Cottrell, 2002). This explains why all attempts to describe rate-independent plasticity by smooth "state variables" were unsuccessful: the trajectories of the actual state variables turn out to be non-differentiable in any point. The situation is similar in classical brittle fracture, where the dissipation is controlled only by the number of broken bonds (Rice, 1978).

To illustrate the general ideas we analyzed in some detail a simple mechanical system whose microscopic dynamics is of a gradient flow type and whose homogenized macroscopic behavior is rate-independent plastic. The basis of the model is a one-dimensional lattice of interacting elastic elements with non-convex energies; the analytic transparency is due to the (mean field type) assumption that the elements interact exclusively through the average strain. The main conceptual limitation of the model is its low-dimensionality and the neglect of disorder. The uneven distribution of the elemental metastability thresholds would produce hardening Sethna et al. (1993); Puglisi and Truskinovsky (2002a) and replace the 
regular stick slip motion with random distribution of avalanches and self-organized criticality (Moretti et al., 2004; Bharathi et al., 2001).

\section{Acknowledgements}

The authors would like to thank J.Rice and A.Larkin for helpful suggestions and to a reviewer for useful references. This work was supported by the research project Cofin-MURST 2004 "Mathematical Models for Material Science" (GP) and by the NSF grant DMS-0102841 (LT).

\section{References}

Abeyaratne, R., Chu, C., James, R.D., 1996. Kinetics of materials with wiggly energies: theory and applications to the evolution of twinning microstructures in a $\mathrm{Cu}-\mathrm{Al}-\mathrm{Ni}$ shape memory alloy. Philos. Mag. A 73 (2), 457-497.

Anzellotti, G., Luckhaus, S., 1987. Dynamic evolution of elasto-plastic bodies. Appl. Math. Optim. 15, 121-140.

Arnol'd, V.I., 1994. Dynamical Systems V. Springer, Berlin.

Balk, A.M., Cherkaev, A.V., Slepyan, L.I., 2001. Dynamics of solids with non-monotone stress-strain relations. 1. Model and numerical experiments. J. Mech. Phys. Solids 49 (1), 131-148.

Ball, J.M., Knops, R.J., Marsden, J.E., 1978. Two Examples in Non-linear Elasticity, Springer Lecture Notes in Mathematics, vol. 665, pp. 41-48.

Berdichevsky, V.L., 2003. Structure of equations of macrophysics. Phys. Rev. E 68, 066126.

Bertotti, G., 1996. Energetic and thermodynamic aspects of hysteresis. Phys. Rev. Lett. 76 (10), $1739-1742$.

Bever, M.B., Holt, D.L., Titchener, A.L., 1972. The stored energy of cold work. Prog. Mater. Sci. 17, $1-190$.

Bharathi, M.S., Lebyodkin, M., Ananthakrishna, G., Fressengeas, C., Kubin, L.P., 2001. Multifractal bursts in the spatiotemporal dynamics of jerky flow. Phys. Rev. Lett. 87(16), 165508-12.

Bhattacharia, K., 1999. Phase boundary propagation in a heterogeneous body. Proc. R. Soc. London A 455, 756-766.

Bridgman, P.W., 1950. The thermodynamics of plastic deformation and generalized entropy. Rev. Mod. Phys. 22 (1), 56-63.

Carpio, A., Bonilla, L.L., 2003. Depinning transitions in discrete reaction-diffusion equations. SIAM J. Appl. Math. 63, 1056-1082.

Cardin, F., Favretti, M., 2003. Dynamics of a chain of springs with non convex potential energy. Math. Mech. Solids 8, 651-669.

Choksi, R., Del Piero, G., Fonseca, I., Owen, D.R., 1999. Structural deformations as energy minimizers in models of fracture and hysteresis. Math. Mech. Solids 4, 321-356.

Cottrell, A.H., 2002. A brief view of work hardening. In: Nabarro, F.R.N., Duesbery, M.S. (Eds.), Dislocations in Solids. Elsevier Science, Amsterdam, pp. vii-xvii.

Deseri, L., Owen, D., 2002. Energetics of two level shears and hardening of single crystals. Math. Mech. Solids 7, 113-147.

Ericksen, J.L., 1975. Equilibrium of bars. J. Elasticity 5, 191-201.

Faciu, C., Suliciu, I., 1994. A Maxwellian model for pseudoelastic materials. Scripta Metall. Mater. 31 (10), 1399-1404.

Fedelich, B., Zanzotto, G., 1992. Hysteresis in discrete systems of possibly interacting elements with a double-well energy. J. Nonlinear Sci. 2, 319-342. 
Fisher, D.S., 1985. Sliding charge-density waves as a dynamic critical phenomenon. Phys. Rev. B 31, 1396-1427.

Fisher, D.S., 1998. Collective transport in random media: from superconductors to earthquakes. Phys. Rep. 301, 113-150.

Heslot, F., Baumberger, T., Perrin, P., Caroli, B., Caroli, C., 1994. Creep stick-slip and dry friction dynamics: experiment and a heuristic model. Phys. Rev. E 49 (6), 4973-4988.

Kestin, J., Rice, J.R., 1970. Paradoxes in the application of thermodynamics to strained solids. In: Stuart, E.G., Gal-Or, B., Brainard, A.J. (Eds.), A Critical Review of Thermodynamics. Mono Book Corp., Baltimore, pp. 275-298.

Kresse, O., Truskinovsky, L., 2004. Lattice friction for crystalline defects: from dislocations to cracks. J. Mech. Phys. Solids 52 (11), 2521-2543.

Lubliner, J., 1998. Plasticity Theory. Prentice Hall, Inc., Englewood Cliffs, NJ.

Mayergoyz, I.D., 1991. Mathematical Models of Hysteresis. Springer, New York.

Menon, G., 2002. Gradient systems with wiggly energies and related averaging problems. Arch. Ration. Mech. Anal. 162, 193-246.

Mielke, A., Theil, F., Levitas, V.I., 2002. A variational formulation of rate-independent phase transformations using an extremum principle. Arch. Ration. Mech. Anal. 162, 137-177.

Moretti, P., Carmen Miguel, M., Zaiser, M., Zapperi, S., 2004. Depinning transition of dislocation assemblies: pileups and low grain boundaries. Phys. Rev. B 69, 214103/1-11.

Müller, I., Villaggio, P., 1977. A model for an elastoplastic body. Arch. Ration. Mech. Anal. 65, 25-46.

Onsager, L., Machlup, S., 1953. Fluctuations and irreversible processes. Phys. Rev. 91, 1505-1512.

Ortiz, M., 1999. Plastic yielding as a phase transition. J. Appl. Mech. 66, 289-298.

Ponter, A.R.S., Bataille, J., Kestin, J., 1979. A thermodynamical model for the time dependent plastic deformation of solids. J. Mécanique 511-539.

Prandtl, L., 1928. Gedankenmodel zur kinetischen theorie der festen koerper. Z. Angew. Math. Mech. 8, $85-106$.

Puglisi, G., Truskinovsky, L., 2000. Mechanics of a discrete chain with bi-stable elements. J. Mech. Phys. Solids 1, 1-27.

Puglisi, G., Truskinovsky, L., 2002a. A mechanism of transformational plasticity. Continnum Mech. Therm. 14, 437-457.

Puglisi, G., Truskinovsky, L., 2002b. Rate independent hysteresis in a bi-stable chain. J. Mech. Phys. Solids 50 (2), 165-187.

Rice, J.R., 1970. On the structure of stress-strain relations for time-dependent plastic deformation in metals. J. Appl. Mech. 37, 728-737.

Rice, J.R., 1975. Continuum mechanics and thermodynamics of plasticity in relation to microscale deformation mechanisms. In: Argon, A.S. (Ed.), Constitutive Equations in Plasticity. MIT Press, Cambridge, MA, pp. 23-79.

Rice, J.R., 1978. Thermodynamics of the quasi-static growth of griffith cracks. J. Mech. Phys. Solids 26, 61-78.

Risken, H., 1989. The Fokker-Plank Equation. Springer, Berlin.

Rosakis, P., Knowles, J.K., 1999. Continuum models for irregular phase boundary motion in shapememory tensile bars. Eur. J. Mech. A. Solids 18, 1-16.

Rosakis, P., Rosakis, A.J., Ravichandran, G., Hodowany, J., 2000. A thermodynamic internal variable model for the partition of the plastic work into heat and stored energy in metals. J. Mech. Phys. Solids 48 (3), 581-607.

Sethna, J.P., Dahmen, K., Kartha, S., Krumhansl, J.A., Woberts, B., Shore, J.D., 1993. Hysteresis and hierarchies: dynamics of disorder-driven first order phase transformations. Phys. Rev. Lett. 70, $3347-3350$.

Suquet, P., 1981. Evolution problems for a class of dissipative materials. Q. Appl. Math. 38, 391-414.

Truskinovsky, L., Vaichtein, A., 2003. Peiers-Nabarro landscape for martensitic phase transitions. Phys. Rev. B 67, 172103-1,4.

Truskinovsky, L., Vaichtein, A., 2004a. Explicit kinetic relation from first principles. In: Gao, D.Y., Ogden, R.W. (Eds.), Advances in Mechanics and Mathematics. Kluwer Academic Publishers, Dordrecht. 
Truskinovsky, L., Vaichtein, A., 2004b. The origin of nucleation peak in transformational plasticity. J. Mech. Phys. Solids 52, 1421-1446.

Vainchtein, A., Rosakis, P., 1999. Hysteresis and stick slip motion of the interfaces in dynamic models of phase transitions. J. Nonlinear Sci. 9 (6), 697-719.

Zaiser, M., Seeger, A., 2002. Long-range internal stresses, dislocation patterning and work hardenning in crystal plasticity. In: Nabarro, F.R.N., Duesbery, M.S. (Eds.), Dislocations in Solids. Elsevier, Amsterdam. 